\title{
Add life to years: psychosocial interventions for people with cognitive disorders*
}

As population longevity increases, living with cognitive disorders during a significant part of the remaining lifespan becomes a strong possibility for the majority of older adults. Therefore, the development of treatment strategies to address the principal causes of cognitive and functional decline is a healthcare priority. The aims of medical and psychological therapies vary according to clinical severity. In the stages of mild cognitive impairment and early dementia, maintaining personal independence and enjoyment of life is a major objective from individual, societal, and economical perspectives (Neugroschl and Wang, 2011; Mokhtari et al., 2012). Current anti-dementia drugs contribute to this goal to a very limited extent. In individuals with mild cognitive impairment these compounds do not prevent the onset of dementia (Raschetti et al., 2007), and in those diagnosed with early dementia, these compounds delay the worsening of symptoms only for several months (Kurz and Lautenschlager, 2010). Drugs with novel mechanisms of action are being developed for Alzheimer's disease and other neurodegenerative conditions. These have the potential to slow down the progression of pathology and expand the number of years for which patients remain at mild levels of cognitive and functional impairment. It is less likely that these treatments will stop cognitive and functional decline completely (Kurz and Perneczky, 2011). Therefore, psychosocial interventions are urgently needed in addition to current and future pharmacotherapy. At any level of cognitive and functional impairment the role of psychosocial interventions is to strengthen and make the best use of preserved skills in order to attain or maintain optimal levels of activity, participation, and interpersonal relationships. In contrast to drugs, psychosocial treatments primarily operate at the behavioral level. Some attempt to restore functions through cognitive, social, or physical activity, while others attempt to minimize disability through compensatory cognitive, behavioral, or environmental strategies. Recent research

\footnotetext{
* The authors wish to dedicate this Guest Editorial to Professor Dr. Hans Lauter on the occasion of his 85 th birthday. As one of the first clinicians and researchers, he promoted the development and evaluation of psychosocial interventions for people with dementia and their family carers.
}

has shown that psychosocial interventions may increase regional cortical thickness or modify the pattern of neuronal network activation (Draganski and May, 2008; van Paasschen et al., 2009; Voss et al., 2010; Erickson et al., 2011). These neurobiological effects clearly demonstrate that brain plasticity persists into old age, but the relationship to behavioral gains and the importance for the prevention of cognitive decline (Lautenschlager et al., 2012; Steffener and Stern, 2012; Valenzuela et al., 2012) are currently unclear.

In recent years psychosocial interventions for people with early-stage dementia have become a growing focus of research, and advances have been made in determining their scope and limitations. Cognitive activity, including stimulation, training, and reminiscence (Woods et al., 2008; Kurz and Lautenschlager, 2011; Woods et al., 2012), as well as physical activity (Cheng et al., 2012; Winchester et al., 2013), provides benefits for memory and other cognitive functions. These effects may outlast the intervention period according to some studies, but in the majority of trials they fade once the treatment is discontinued. Moreover, whereas most studies have not assessed real-life outcomes, improvements in the participants' ability to manage day-to-day problems have been reported in a handful of studies (Graff et al., 2007; Clare et al., 2010; Gitlin et al., 2010). It is important to note that many studies conducted thus far to demonstrate the efficacy of psychosocial interventions in people with cognitive disorders had significant methodological limitations which may have reduced their ability to capture treatment-related changes.

To reveal the true potential of psychosocial interventions, more and better research is needed. Future studies should increase the field's scientific rigor by introducing improvements of design over previous trials, particularly with regard to sample size, treatment duration, standardization of interventions, transfer of treatment strategies into everyday life, matching of intervention and outcomes, and long-term follow-up (Craig et al., 2008). Such high-quality studies are costly, and unlike in drug trials, industry involvement cannot be expected. Therefore, significant progress can only be made if governments and private foundations allocate 
more resources to research in this important area. The field will also certainly benefit from harmonization of methodology. Although the diversity of theoretical foundations and treatment strategies inevitably entails a broader spectrum of outcomes than in clinical drug trials, it is desirable to assess a common set of efficacy measures which are patient-centered, clinically meaningful, and appropriately validated. In addition to various aspects of cognitive ability, core outcomes should include coping with day-to-day problems, attainment of individually relevant goals, and patient- and carerrated quality of life. Alignment of measures will enable comparisons across studies as well as pooling and meta-analytic evaluation of data. Such methods are required for selecting the most efficacious strategies and strategy combinations, identifying determinants of treatment success, defining optimal treatment dosage and duration, and personalizing psychosocial interventions according to individual needs and capabilities.

While improvements in the design of both interventions and trials evaluating their efficacy are required in future research, it is equally important to consolidate the role of psychosocial interventions in the broader context of the management of cognitive disorders. Evidence should be provided about which strategies are most beneficial at what level of clinical severity. Furthermore, the most efficacious, practical, and cost-effective combinations of psychosocial strategies and drug treatments, as well as the added value of such combinations over monotherapy, need to be identified.

A particularly promising yet largely unexplored strategy to support autonomy and quality of life in people with cognitive disorders is the use of modern information and communication technology, including sensor systems, smart phones, and tablet computers. Such aids can be used for a variety of purposes such as providing reminders of daily tasks, guiding activities, maintaining social links, supporting navigation outside the home, monitoring physiological functions, and ensuring safety of household appliances (Mokhtari et al., 2012). Technology devices may also be an elegant and effective way to implement psychosocial strategies in everyday living. These strategies will attain growing importance in the future as the digital literates are coming of age (Bharucha et al., 2009).

The ultimate goal of research in this field is to establish psychosocial interventions alongside pharmacological treatment as a strong component in the management of cognitive disorders. It is desirable for clinicians to offer patients the complementary benefits from both approaches. Patients should be able to take advantage of pharmacotherapy in order to gain years of relatively preserved cognitive and functional ability, and they might use psychosocial approaches to replenish these years with activity, participation, and enjoyable social bonds.

\section{Conflict of interest}

Nicola T. Lautenschlager is the current Editorin-Chief of International Psychogeriatrics. Therefore, one of the deputy editors reviewed this editorial.

\section{AleXANDER KurZ, ${ }^{1}$ LiNDA ClaRE ${ }^{2}$ AND NiCOLA T. LAUTENSCHLAGER ${ }^{3,4}$}

${ }^{1}$ Department of Psychiatry and Psychotherapy, Klinikum rechts der Isar, Technische Universität München, Munich, Germany

${ }^{2}$ Research in Ageing and Cognitive Health, School of Psychology, Bangor University, Gwynedd, UK

${ }^{3}$ Academic Unit for Psychiatry of Old Age, St Vincent's Health, Department of Psychiatry, University of Melbourne, Parkville, Victoria, Australia

${ }^{4}$ School of Psychiatry \& Clinical Neurosciences \&

Western Australian Centre for Health \& Ageing, University of Western Australia, Crawley WA, Perth, Australia

Email: alexander.kurz@lrz.tum.de

\section{References}

Bharucha, A. J. et al. (2009). Intelligent assistive techology applications to dementia care: current capabilities, limitations and future challenges. American fournal of Geriatric Psychiatry, 17, 88-104.

Cheng, S. T., Chow, P. K., Yu, E. C., Chan, A. C., Lee, T. M. and Lam, J. H. (2013, Feb 6). Mental and physical activities delay cognitive decline in older persons with dementia. American fournal of Geriatric Psychiatry.

Clare, L. et al. (2010). Goal-oriented cognitive rehabilitation for people with early-stage Alzheimer disease: a single-blind randomized controlled trial of clinical efficacy. American Fournal of Geriatric Psychiatry, 18, 928-939.

Craig, P., Dieppe, P., Macintyre, S., Michie, S., Nazareth, I. and Petticrew, M. (2008, Sep 29). Developing and evaluating complex interventions: the new Medical Research Council guidance. BMF, doi:10.1136/bmj.a1655.

Draganski, B. and May, A. (2008). Training-induced structural changes in the adult human brain. Behavioural Brain Research, 192, 137-142.

Erickson, K. I. et al. (2011). Exercise training increases size of hippocampus and improves memory. Proceedings of the National Academy of Sciences, 108, 3017-3022.

Gitlin, L. N., Winter, L., Hodgson, M. P. D. and Hauck, W. W. (2010). A biobehavioral home-based intervention and the well-being of patients with dementia and their caregivers. The COPE randomized trial. $\mathcal{F A M A}, 304$, 983-991.

Graff, M., Vernooij-Dassen, M. J., Thijssen, M., Dekker, J., Hoefnagels, W. H. and Olde-Rikkert, M. G. (2007). 
Effects of community occupational therapy on quality of life, mood and health status in dementia patients and their caregivers: a randomized controlled trial. Fournals of Gerontology Series A, 62A, 1002-1009.

Kurz, A. F. and Lautenschlager, N. L. (2010). Established treatments for Alzheimer's disease: cholinesterase inhibitors and memantine. In D. Ames, A. Burns and J. O'Brien (eds.), Dementia (pp. 471-483). London: Hodder Arnold.

Kurz, A. F. and Lautenschlager, N. T. (2011). The clinical significance of cognition-focused interventions for cognitively impaired older adults. International Psychogeriatrics, 23, 1364-1375.

Kurz, A. and Perneczky, R. (2011). Novel insights for the treatment of Alzheimer's disease. Progess in Neuro-Psychopharmacology and Biological Psychiatry, 35, 373-379.

Lautenschlager, N. T., Cox, K. and Cyarto, E. V. (2012). The influence of exercise on brain aging and dementia. Biochimica et Biophysica Acta, 1822, 474-481.

Mokhtari, M., Aloulou, H., Tiberghien, T., Biswas, J. and Racoceanu, D. (2012). New trends to support independence in persons with mild dementia - a mini-review. Gerontology, 58, 554-563.

Neugroschl, J. and Wang, S. (2011). Alzheimer's disease: diagnosis and treatment across the spectrum of disease severity. Mount Sinai fournal of Medicine, 78, 596-612.
Raschetti, R., Albanese, E., Vanacore, N. and Maggini, M. (2007). Cholinesterase inhibitors in mild cognitive impairment: a systematic review of randomised trials. PLoS Medicine, 4, 1818-1828.

Steffener, J. and Stern, Y. (2012). Exploring the neural basis of cognitive reserve in aging. Biochimica and Biophysica Acta, 1822, 467-473.

Valenzuela, M. J. et al. (2012). Multiple biological pathways link cognitive lifestyle to protection from dementia. Biological Psychiatry, 71, 783-791.

Van Paasschen, J., Clare, L., Woods, R. T. and Linden, E. E. J. (2009). Can we change brain functioning with cognition-focused interventions in Alzheimer's disease? The role of functional neuroimaging. Restorative Neurology and Neuroscience, 27, 473-491.

Voss, M. W. et al. (2010). Plasticity of brain networks in a randomized intervention trial of exercise training in older adults. Frontiers in Aging Neuroscience, 2, 1-17.

Winchester, J. et al. (2013). Walking stabilizes cognitive functioning in Alzheimer's disease (AD) across one year. Archives of Gerontology and Geriatrics, 56, 96-103.

Woods, B., Aguirre, E., Spector, A. E. and Orrell, $M$. (2012). Cognitive stimulation to improve cognitive functioning in people with dementia (review). Cochrane Database of Systematic Reviews, CD005562.

Woods, B., Spector, A., Jones, C., Orrell, M. and Davies, S. (2008). Reminiscence therapy for dementia. Cochrane Database of Systematic Reviews, CD001120. 